\title{
IOA: Improving SVM Based Sentiment Classification Through Post Processing
}

\author{
Peijia Li, Weiqun Xu, Chenglong Ma, Jia Sun, Yonghong Yan \\ The Key Laboratory of Speech Acoustics and Content Understanding \\ Institute of Acoustics, Chinese Academy of Sciences \\ No. 21 North 4th Ring West Road, Haidian District, 100190 Beijing, China \\ \{lipeijia, xuweiqun, machenglong, sunjia, yanyonghong\} @hccl.ioa.ac.cn
}

\begin{abstract}
This paper describes our systems for expression-level and message-level sentiment analysis - two subtasks of SemEval-2015 Task 10 on sentiment analysis in Twitter. First we built two baseline systems for the two subtasks using SVM with a variety of features. Then we improved the systems through model iteration and probability-output weighting respectively. Our submissions are ranked the 3rd and 2nd among eleven teams on the 2015 test set and progress test set in subtask A and the 7th and 4th among 40 teams on the two test sets respectively in subtask $\mathrm{B}$.
\end{abstract}

\section{Introduction}

Recently sentiment analysis has become one of the most popular research topics in the natural language processing community, mainly due to the exponential growth of social media data replete with subjective information. The once neglected topic has spurred immense interests from both academia and industry. Many approaches have been proposed for sentiment analysis in customer reviews, blogs and microblogs (for good reviews, see (Pang and Lee, 2008; Liu, 2012; Kiritchenko et al., 2014)). These approaches can be roughly divided into two categories. One is knowledge intensive or rule-based approaches, e.g., (Taboada et al., 2011; Reckman et al., 2013). Such approaches can achieve reasonably good results when tailored for a specific domain but their maintainability and cross domain portability is usually weak. The other is data intensive or machine learning-based, which learns to analyse sentiment from data. It is currently the most predominant approach, including supervised learning, deep learning etc. Sentiment analysis is often taken as a classification task. Widely used classifiers include Support Vector Machines (SVM), Maximum Entropy Models (MaxEnt), and naive Bayes classifiers. Common features include word/character n-grams and sentiment lexicons, among others. Key research issues for learning approaches include feature engineering, model selection, ensemble learning, etc.

SemEval 2015 task10 (Rosenthal et al., 2015) is a sequel to the two tasks on sentiment analysis in Twitter in the past two years (Nakov et al., 2013; Rosenthal et al., 2014). They have provided freely available, annotated corpus as a common testbed and significantly promoted sentiment analysis in tweetlike short and informal texts. The same metric, i.e., the average $F_{1}$ score of positive and negative classes, is used for measuring performances. But this year there are some changes. Besides the classical expression-level (A) and message-level (B) subtasks, another three subtasks are added, i.e., subtask $\mathrm{C}$ - topic-based message polarity classification, subtask D - detecting trends towards a topic, and subtask E - determining strength of association of twitter terms with positive sentiment. The organisers make no distinction between constrained and unconstrained systems, which means participants could utilise any other data. But it has to be described in the submission form.

We submitted systems only for the expressionlevel and message-level subtasks. In this paper, we provide some details behind the systems. 


\begin{tabular}{|c|r|r|}
\hline Data & TaskA & TaskB \\
\hline Twittter2013-train & 7,639 & 7,972 \\
Twittter2013-dev & 929 & 1,372 \\
Twittter2013-test & 3,625 & 3,198 \\
SMS2013-test & 2,334 & 2,093 \\
Twittter2014-test & 2,028 & 1,561 \\
LiveJournal2014-test & 1,315 & 1,142 \\
Sarcasm2014-test & 124 & 86 \\
\hline Twitter2015-test & 3,092 & 2,390 \\
Progress2015-test & 10,681 & 8,987 \\
\hline
\end{tabular}

Table 1: Statistics of all the datasets. The last row of Progress2015-test data is composed of all the previous test data sets.

\section{Our System}

Our systems are built with an SVM classifier using various features and resources, including sentiment lexicons and word vectors. To further improve the performance, we use model iteration and probability-output weighting.

\subsection{Resources}

The resources used in our system are as follows:

Labeled training and test data: Although the organisers make no difference between constrained and unconstrained systems, it is not easy to make additional data effective (Rosenthal et al., 2014). So we just use the provided labeled data. However, since we did not participate in the past two evaluations, we are unable to get the full labeled data because some tweets are unavailable. But we crawled as much data as possible using the provided script. Table 1 shows the size of the labeled data and test data we get. The 2015 test data is released directly and the results are required to be submitted in one week. We take the training data and development data as our training data. The test data from the previous years can be used for tuning parameters (but NOT for training).

Sentiment Lexicons and Word Embedding: As many researchers have showed, e.g., (Mohammad et al., 2013), sentiment lexicons play an important role in sentiment analysis. In our system, seven sentiment lexicons are used: the Hashtag Sentiment lexicon, the Sentiment140 lexicon (Mohammad and Turney, 2010), the MPQA lexicon (Wilson et al.,

\begin{tabular}{|c|c|c|}
\hline Feature & subtask A & subtask B \\
\hline word ngrams & $\checkmark$ & $\checkmark$ \\
POS & & $\checkmark$ \\
clusters & & $\checkmark$ \\
word vector & $\checkmark$ & $\checkmark$ \\
negation & $\checkmark$ & $\checkmark$ \\
lexicons & $\checkmark$ & $\checkmark$ \\
characters & $\checkmark$ & \\
\hline
\end{tabular}

Table 2: Features extracted for each subtask.

2005), the Bing Liu lexicon (Hu and Liu, 2004), the AFINN-111 (Nielsen, 2011), the SentiWordNet (Baccianella et al., 2010) and the Hedonometer lexicon $^{1}$. In addition, as word embeddings have been utilised to produce promising results in various NLP applications, we use sentiment-specific word embedding (Tang et al., 2014) in our system.

LibSVM: We used the package LibSVM (Chang and Lin, 2011) to construct the classification model for both subtasks.

CMU Tweet NLP: It is an open resource (Owoputi et al., 2013) for analysing tweets and was used to extract features for tokenising, POS tagging and clustering.

\subsection{Preprocessing}

The main preprocessing steps are the following:

- All upper case letters are converted to lower case ones

- URLs and user names are replaced with strings ‘http://someurl' and '@someuser' respectively

- Tokenise and label the tweets with partof-speech using Carnegie Mellon University (CMU) tool (Owoputi et al., 2013)

\subsection{Features}

After preprocessing, each tweet is represented as a feature vector made up of part of the following features, the features used in each subtask are shown in Table 2.

- Word N-grams: A binary value of contiguous n-grams of $1,2,3$, and 4 tokens and noncontiguous $n$-grams $(n=3,4)$. Non-contiguous

\footnotetext{
${ }^{1}$ http://hedonometer.org/words.html
} 
n-grams are those intermediate grams that are replaced with a special symbol like ' $*$ '. For example, a 4-gram "I** guys" is the corresponding non-contiguous gram of contiguous gram "I love you guys".

- Character N-grams: Although character ngrams have been used in sentiment analysis by many researchers, we find that the features are not effective for subtask B, so they are only used for subtask A. This feature is the binary value of the two and three prefix and suffix letters.

- POS: Ten features are added by pos tagging. They are respectively the count of interjection, adverb, preposition, article, verb, punctuation, noun, pronoun, adjective and hashtag in a tweet.

- Clusters: Every token in a tweet is mapped to one of Twitter Word Clusters by CMU tool (Owoputi et al., 2013). The features extracted are a boolean vector showing the presence or absence of the tweet in the 1000 clusters which are generated from about 56 million tweets.

- Word Vector: Words are represented as a vector of 50 dimensions. Then we use min, average and max functions to convert the embeddings into fixed-length features, in a way similar to the pooling technique used in CNN to get a tweet vector representation. So another three features are added.

- Negation: A binary value indicating the negated contexts. The "_NEG" suffix is appended to grams if they are in a negation scope which starts with a negation word and ends with certain punctuation marks ${ }^{2}$.

- Lexicons: For each token in one tweet, if it appears in sentiment lexicons in section 2.1, it is mapped to the corresponding score. In the lexicons which have no sentiment score we set the positive +1 and the negative -1 . Other tokens are set to zero. Then a tweet would be represented with its total score, maximal score,

\footnotetext{
${ }^{2} \mathrm{http}: / /$ sentiment.christopherpotts.net/lingstruc.html\#negation
}

minimal score, negative score, last word score which does not equal zero, and the count of tokens with non-negative score.

\subsection{Training}

SVM is used as the classifier in our systems with the features described in section 2.3. We trained SVM on the labeled tweets with the RBF kernel and tuned the parameters on the dev dataset. For both subtasks, we tuned the parameters for Twitter2015 test data using the Twitter2013, Twitter2014 test data as dev dataset and tuned the parameters for the progress 2015 test data using all the previous test data as dev dataset. The parameters were tuned to maximise the average $F_{1}$ score of positive and negative classes using brute-force grid search.

\subsection{Post-processing}

We tried different strategies for the different subtasks. For subtask A, we adopted a model iteration approach described in Algorithm 1. For subtask B, we used probability-output weighting to adapt SVM model with RBF kernel to the data set, similar to (Miura et al., 2014).

\subsubsection{Model iteration for expression-level subtask}

It was found that utilising more external data did not improve the performance as expected because of the different data resource and annotation method (Rosenthal et al., 2014). So we tried a model iteration approach. ${ }^{3}$ We added the test data labeled with high confidence into the training data and then retrained a new model. The algorithm for subtask A is given in Algorithm 1 and the experiment results are given in section 3.1.

\footnotetext{
${ }^{3} \mathrm{NB}$ : Our approach is different from the semi-supervised learning in that we use limited test data while semi-supervised learning usually uses a large number of external data.
} 


\begin{tabular}{|l|c|c|c|c|c|c|}
\hline Data & $c$ & $g$ & $I$ & $p$ & $w_{\text {pos }}$ & $w_{\text {neg }}$ \\
\hline A-Twitter15 & 1100 & 0.00287 & 2 & 0.8 & - & - \\
A-Progress15 & 1100 & 0.00287 & 2 & 0.8 & - & - \\
B-Twitter15 & 1200 & 0.00267 & - & - & 3.2 & 2.2 \\
B-Progress15 & 1200 & 0.00267 & - & - & 2.1 & 1.4 \\
\hline
\end{tabular}

Table 3: The parameters for different test data. $I$ is the maximum number of iteration. $w_{\text {pos }}$ and $w_{\text {neg }}$ are weight parameters.

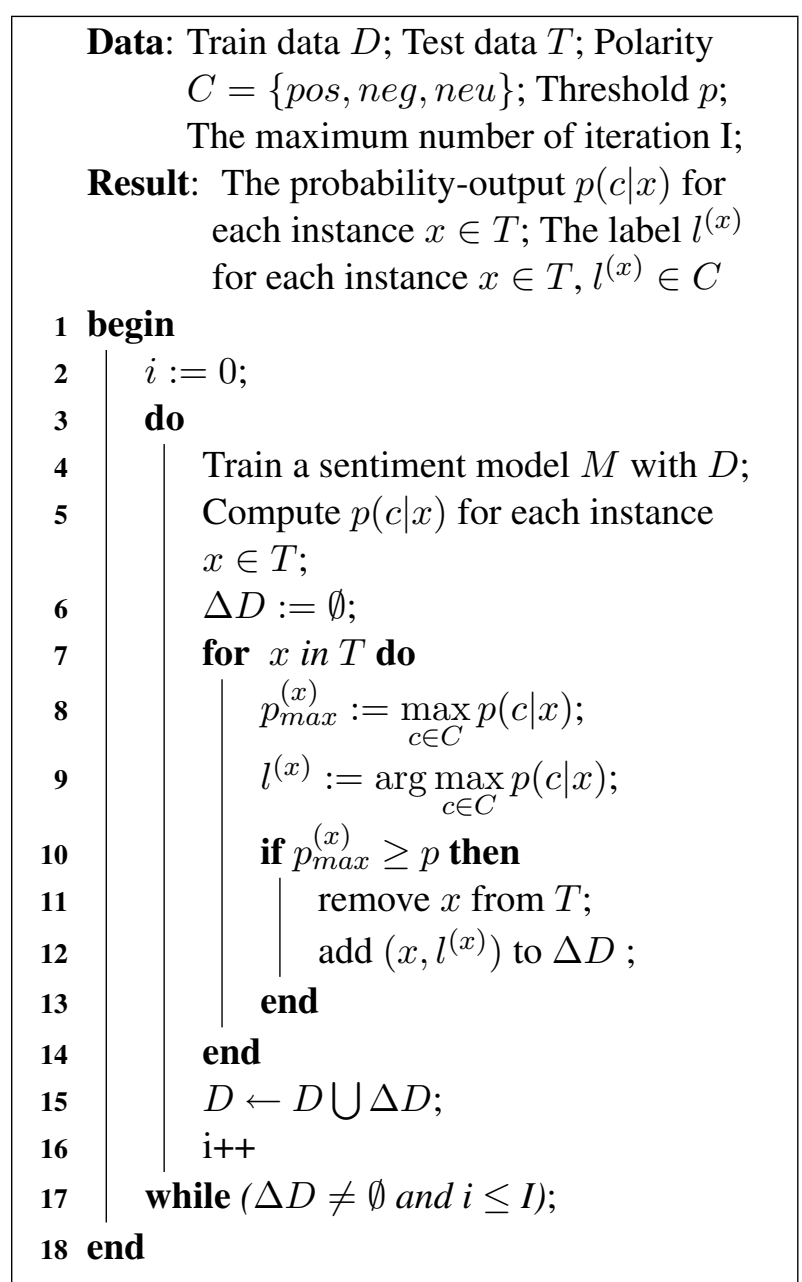

Algorithm 1: Model iteration for subtask A.

\subsubsection{Probability output weighting for message-level subtask}

We applied probability-output weighting (Miura et al., 2014) into SVM and adapted it to subtask B. For a tweet $x$, the base model output probability $p(c \mid x)$ for each polarity $c(c \in\{$ pos, neg,neu $\}$ ). A weighting factor $w_{c}$ that adjusted the probabilityoutput $p(c \mid x)$ was introduced. The system labeled the tweet with polarity $c$ which maximises the prod-

\begin{tabular}{|c|c|c|c|c|}
\hline \multirow{2}{*}{ Data } & \multicolumn{2}{|c|}{ subtask A } & \multicolumn{2}{c|}{ subtask B } \\
\cline { 2 - 5 } & baseline & submitted & baseline & submitted \\
\hline Twtitter15 & 82.31 & 82.76 & 60.02 & 62.62 \\
Twitter13 & 83.86 & 83.90 & 68.79 & 71.32 \\
SMS & 84.38 & 84.18 & 68.03 & 68.14 \\
Twitter14 & 85.09 & 85.37 & 68.70 & 71.86 \\
LiveJounal & 85.47 & 85.62 & 71.68 & 74.52 \\
Sarcasm & 71.81 & 71.81 & 53.70 & 51.48 \\
\hline
\end{tabular}

Table 4: The overall results.

uct of $w_{c}$ and $p(c \mid x)$, namely $\arg \max w_{c} \times p(c \mid x)$. The weighting parameters $w_{c}$ for each polarity was tuned by maximising the accuracy using grid-search in the corresponding dev data. The results can be seen in section 3.2.

\section{Experiments and Results}

The official evaluation metric of the task is the average $F_{1}$ score of the positive and the negative classes. After the base training (Section 2.4), we got the base results in Table 4, "baseline" columns. Then we focused on improving systems for both subtasks. And the improved (or not) results are shown in the "submitted" columns.

\subsection{Subtask A: expression-level sentiment analysis}

We built the system using 8,568 tweets, including 7,639 training tweets and 929 development tweets described in section 2.1 using the features in section 2.3. After the release of the labeled test data, we compared the performance using the same model to rerun the test data. We set different threshold parameters $p$ referred in section 2.5 to compare the results. The experiment results are given in Table 5 . 


\begin{tabular}{|l|c|c|c|c|c|c|c|}
\hline Threshold $p$ & 0.70 & 0.75 & $\mathbf{0 . 8 0}$ & 0.85 & 0.90 & 0.95 & 1.00 \\
\hline Twitter2015 & 82.42 & 82.56 & $\mathbf{8 2 . 7 6}$ & 82.76 & 82.70 & 82.53 & 82.31 \\
Twitter2013 & 83.95 & 84.00 & $\mathbf{8 3 . 9 0}$ & 84.62 & 84.49 & 84.38 & 83.86 \\
SMS & 84.02 & 84.09 & $\mathbf{8 4 . 1 8}$ & 84.41 & 84.43 & 84.48 & 84.38 \\
Twitter2014 & 84.96 & 85.44 & $\mathbf{8 5 . 3 7}$ & 85.13 & 84.81 & 85.17 & 85.09 \\
LiveJounal & 85.58 & 85.31 & $\mathbf{8 5 . 6 2}$ & 85.61 & 85.58 & 85.58 & 85.47 \\
Sarcasm & 71.81 & 71.58 & $\mathbf{7 1 . 8 1}$ & 73.07 & 71.81 & 71.58 & 71.81 \\
\hline
\end{tabular}

Table 5: The results for subtask A under different threshold $p$. Numbers in bold are the submitted results.

\subsection{Subtask B: message-level sentiment analysis}

We adapted the probability-output weighting approach to subtask B. The experiment result shows that weighting is effective for this subtask. The improvement using the parameters in Table 3 can be seen from Table 4.

The approach is effective for improving the twitter $F_{1}$ score but degrades the performance on the Sarcasm data, maybe because it depends too much on the data.

\subsection{Experiment analysis}

For subtask A, we made iteration stop at $i=2$. The reason why there is little improvement is: (1) After each iteration, the number of new data added to the training data for retraining a new model is rather small. (2) Once the classifier puts a high confidence on a label, this instance is very likely to be similar to existing instances, which means the added instances would not contribute very much to classification.

In the experiments after submission, we tried to interchange the improvement method between the subtasks, but they showed a little decrease on both subtasks. When the model iteration approach was used in subtask B, we did not receive expected improvement. This may be because that the performance for subtask B is lower than that for subtask A, which may result in the wrong samples added into the training data. When the probability-output weighting approach was used on subtask A, we only got limited improvement in the $F_{1}$ score.

\section{Conclusion}

We described our system for two subtasks of SemEval 2015 task 10 - Sentiment Analysis in Twitter. Our systems are built by integrating a variety of features into SVM as baselines and then improved by model iteration and probability-output weighting for expression-level and message-level subtasks respectively. We compared the results and analyse the reason of the improvement. Our submissions are ranked the 3rd and 2nd among eleven teams on the 2015 test set and progress test set in subtask A and the 7th and 4th among 40 teams on the two test sets respectively in subtask $\mathrm{B}$.

\section{Acknowledgments}

We would like to thank the shared task organisers for their support throughout this work. This work is partially supported by the National Natural Science Foundation of China (Nos. 11161140319, 91120001, 61271426), the Strategic Priority Research Program of the Chinese Academy of Sciences (Grant Nos. XDA06030100, XDA06030500), the National 863 Program (No. 2012AA012503) and the CAS Priority Deployment Project (No. KGZDEW-103-2).

\section{References}

Stefano Baccianella, Andrea Esuli, and Fabrizio Sebastiani. 2010. SentiWordNet 3.0: An enhanced lexical resource for sentiment analysis and opinion mining. In LREC, volume 10, pages 2200-2204.

Chih-Chung Chang and Chih-Jen Lin. 2011. LIBSVM: a library for support vector machines. ACM Transactions on Intelligent Systems and Technology (TIST), 2(3):27.

Minqing $\mathrm{Hu}$ and Bing Liu. 2004. Mining and summarizing customer reviews. In Proceedings of the tenth ACM SIGKDD international conference on Knowledge discovery and data mining, pages 168-177.

Svetlana Kiritchenko, Xiaodan Zhu, and Saif M. Mohammad. 2014. Sentiment analysis of short infor- 
mal texts. Journal of Artificial Intelligence Research (JAIR), 50:723-762.

Bing Liu. 2012. Sentiment analysis and opinion mining. Synthesis Lectures on Human Language Technologies, 5(1):1-167.

Yasuhide Miura, Shigeyuki Sakaki, Keigo Hattori, and Tomoko Ohkuma. 2014. Teamx: A sentiment analyzer with enhanced lexicon mapping and weighting scheme for unbalanced data. In Proceedings of the 8th International Workshop on Semantic Evaluation (SemEval 2014), pages 628-632, Dublin, Ireland, August.

Saif M Mohammad and Peter D Turney. 2010. Emotions evoked by common words and phrases: Using mechanical turk to create an emotion lexicon. In Proceedings of the NAACL HLT 2010 Workshop on Computational Approaches to Analysis and Generation of Emotion in Text, pages 26-34.

Saif Mohammad, Svetlana Kiritchenko, and Xiaodan Zhu. 2013. Nrc-canada: Building the state-of-theart in sentiment analysis of tweets. In Second Joint Conference on Lexical and Computational Semantics (*SEM), Volume 2: Proceedings of the Seventh International Workshop on Semantic Evaluation (SemEval 2013), pages 321-327, Atlanta, Georgia, USA, June.

Preslav Nakov, Sara Rosenthal, Zornitsa Kozareva, Veselin Stoyanov, Alan Ritter, and Theresa Wilson. 2013. Semeval-2013 task 2: Sentiment analysis in Twitter. In Second Joint Conference on Lexical and Computational Semantics (*SEM), Volume 2: Proceedings of the Seventh International Workshop on Semantic Evaluation (SemEval 2013), pages 312-320, Atlanta, Georgia, USA, June.

Finn Årup Nielsen. 2011. A new ANEW: evaluation of a word list for sentiment analysis in microblogs. In Proceedings of the ESWC2011 Workshop on 'Making Sense of Microposts': Big things come in small packages, Heraklion, Crete, Greece, May 30, 2011, pages 93-98.

Olutobi Owoputi, Brendan O'Connor, Chris Dyer, Kevin Gimpel, Nathan Schneider, and Noah A. Smith. 2013. Improved part-of-speech tagging for online conversational text with word clusters. In $H L T-N A A C L$, pages 380-390.

Bo Pang and Lillian Lee. 2008. Opinion mining and sentiment analysis. Foundations and trends in information retrieval, 2(1-2):1-135.

Hilke Reckman, Cheyanne Baird, Jean Crawford, Richard Crowell, Linnea Micciulla, Saratendu Sethi, and Fruzsina Veress. 2013. teragram: Rule-based detection of sentiment phrases using sas sentiment analysis. In Second Joint Conference on Lexical and Computational Semantics (*SEM), Volume 2: Proceedings of the Seventh International Workshop on Semantic
Evaluation (SemEval 2013), pages 513-519, Atlanta, Georgia, USA, June.

Sara Rosenthal, Alan Ritter, Preslav Nakov, and Veselin Stoyanov. 2014. Semeval-2014 task 9: Sentiment analysis in Twitter. In Proceedings of the 8th International Workshop on Semantic Evaluation (SemEval 2014), pages 73-80, Dublin, Ireland, August.

Sara Rosenthal, Preslav Nakov, Svetlana Kiritchenko, Saif M Mohammad, Alan Ritter, and Veselin Stoyanov. 2015. Semeval-2015 task 10: Sentiment analysis in Twitter. In Proceedings of the 9th International Workshop on Semantic Evaluation, SemEval '2015, Denver, Colorado, June.

Maite Taboada, Julian Brooke, Milan Tofiloski, Kimberly D. Voll, and Manfred Stede. 2011. Lexiconbased methods for sentiment analysis. Computational Linguistics, 37(2):267-307.

Duyu Tang, Furu Wei, Bing Qin, Ming Zhou, and Ting Liu. 2014. Building large-scale Twitter-specific sentiment lexicon : A representation learning approach. In COLING 2014, 25th International Conference on Computational Linguistics, Proceedings of the Conference: Technical Papers, August 23-29, 2014, Dublin, Ireland, pages 172-182.

Theresa Wilson, Janyce Wiebe, and Paul Hoffmann. 2005. Recognizing contextual polarity in phrase-level sentiment analysis. In Proceedings of the conference on human language technology and empirical methods in natural language processing, pages 347-354. 